\title{
Identification of Microphases: New Databases for Electron Diffraction
}

\author{
H.V. Hart and D.R. Denley
}

Shell Chemical LP, P.O. Box 1380, Houston, TX 77251-1380, USA

We have developed the methodology for rapid identification of minute samples of unknown material (as little as ca. $10^{-16}$ gram) through the technique of selected area electron diffraction. This can be applied either to samples that are too small to be practical for traditional x-ray diffraction (XRD), or for the case where the phase of interest is isolated in a much larger amount of some other matrix that may interfere with collection of XRD patterns.

Search/match procedures for polycrystalline electron diffraction have been previously reviewed [1], and, absent a unit cell determination, the only widely available and comprehensible database is the NIST/Sandia/ICDD Electron Diffraction Database [2], queried through a program known as EDSEARCH. If a unit cell can be determined, the National Institute for Standards and Technology (NIST) Crystal Data [3] can be searched directly with the reduced unit cell parameters.

As noted by previous researchers on this subject, a major disadvantage of using any version of the PDF (which is based on X-ray diffraction) is that some d-spacings measured by electron diffraction are the result of double diffraction only, and are therefore not observed in X-ray diffraction or included in the PDF. Unfortunately, there is no existing comprehensive database for electron diffraction which utilizes d-spacing search/match (as opposed to unit cell searching) and which rigorously incorporates double diffraction. As seen below, rigorous treatment is possible by calculating dspacings from reduced unit cells, conveniently available in NIST Crystal Data. In this way, all experimentally observable d-spacings are contained in the database, and no database d-spacing is impossible to be observed experimentally (weak intensities not included).

Additionally, in today's PC environment it is important to use familiar desktop productivity tools including database, flexible reporting, ease of transfer and reformatting of data, and ease of interpretation of output.

Microanalysis in the transmission electron microscope (TEM) involves interpreting morphology via density variations (manifested by differences in electron absorption) and $\mathrm{x}$ ray elemental analysis (similar to x-ray fluorescence for bulk samples). A third technique, electron diffraction, is less common, is applicable to crystalline materials only, and involves matching a diffraction pattern against that of known materials. Because available databases are cumbersome to use or only partly successful, two new databases were built for a PC with Microsoft ACCESS 97 from the National Institute for Standards and Technology (NIST) Crystal Data. RINGS [4] is for polycrystalline samples, which exhibit concentric circles in electron diffraction mode, and ZONES [5], is for single crystals, which show spot patterns. Both databases incorporate a total of 79,136 inorganic phases. 
Features of RINGS include: 1) More rigorous treatment of double diffraction to help eliminate poor matches. 2) Experimental error limits are adjustable to fit the capabilities of current instrumentation. 3) The Figure of merit (FOM) has been improved to include the number of experimental d-spacings that match the database, but also to discount for failures of database d-spacings, within the experimental range, which have no match. This is very critical to eliminating false matches to phases with large and/or low symmetry unit cells, for which there may be coincidental matches to experimental values, but also many unmatched database d-spacings.

A second database, ZONES, for single crystal electron diffraction was developed, where the two largest d-spacings and their interangle are matched to calculated values for common zones. Again, NIST Crystal Data reduced unit cells are used to construct this database for reasons related to double diffraction as described above.

ZONES is a relational database built from NIST Crystal Data for the identification of single crystals by selected area electron diffraction (SAED) and elemental analysis. The two largest experimental d-spacings and included angle in a zone are matched against values calculated from reduced unit cells, thereby fully and rigorously incorporating the effects of double diffraction. This and inclusion of elemental information to limit the possible solutions to a manageable number yield a robust approach. Indexing the photograph is unnecessary and is not an identification tool.

ZONES contains up to 25 zones (calculated $\mathrm{d}_{1}, \mathrm{~d}_{2}, \varphi$ ) per phase plus coded elemental analysis above $Z=10$. It was successful in identifying a wide variety of phases from zone axis SAED and EDS data. Successful searches employed test data obtained experimentally and from the literature, with errors up to $4 \%$ on $d$ and 2 deg on $\varphi$.

ZONES has all the robustness of a reduced unit cell search, but requires simpler input and no knowledge of crystallography to use. In certain cases of isomorphism or polymorphism, additional, related solutions may reveal structural relationships not apparent from unit cell comparisons alone.

\section{References}

[1] R. Anderson et al., Micros. Soc. Amer. Bull. 23(1), (1993) 128-137; A. Mighell, \& V. L. Karen, J. Res. Nat. Inst. Std. Tech. 101(3), (1996). 273-280)

[2] M. J. Carr, et al., J. Res. Nat. Inst. Std. Tech. 94(1), (1989) 15-20.

[3] J. K. Stalick, \& A. D. Mighell, Crystal Data Version 1.0 Database Specifications. NBS Technical Note 1229. (1986) Washington, DC: National Bureau of Standards.

[4] D. R. Denley and H. V. Hart, J. Appl. Cryst. 35, (2002) 546-551

[5] H. V. Hart, J. Appl. Cryst. 35, (2002) 552-555 